\title{
Process for Physicochemical Improvement of Ultra- flexible Liposomes Loaded with Insulin
}

\author{
S. M. ARCINIEGAS, M. J. BERNAD¹, S. C. CABALLERO, D. HERNANDEZ², B. SOLIS ${ }^{2}$ AND D. VARGAS*
}

Physiology and Pharmacology Department, Veterinary Medicine School, ${ }^{1}$ Pharmaceutical Technology Chemistry School, Universidad Nacional Autónoma de México, Ciudad Universitaria, Universidad 3000, Circuito Exterior S/N Delegación Coyoacán, C.P. 04510, ²Laboratory of Pharmaceutical Assays, Facultad de Estudios Superiores (FES) - Cuautitlán, Universidad Nacional Autónoma de México, Carretera Cuautitlán-Teoloyucan Km. 2.5, Col. San Sebastián Xhala, Cuautitlán Izcalli, Estado de México, CP. 54714, México

Arciniegas et al.: Manufacturing method for liposomes with high insulin load

\begin{abstract}
The objective of this work was to evaluate physicochemical characteristics of ultra-flexible liposomes obtained by two manufacturing methods with sucrose as hydrating agent, the thin-film rehydration method and the heating method, with a focus on improvement of percent entrapped insulin. Four formulations were prepared by each method with different percentages of soy L-a-phosphatidylcholine and sodium cholate. Size distribution, polydispersity index, zeta potential and encapsulation percentage were evaluated. The ultraflexible liposomes obtained using the heating method showed smaller size, better homogeneity, adequate surface charge and higher encapsulation percent compared with traditional liposomes and those obtained using the thin-film rehydration method. Additionally, insulin was found to be stable after the formulation processes according to polyacrylamide gel electrophoresis and differential calorimetry scanning, indicating that the temperature used in these techniques did not affect the stability of this protein.
\end{abstract}

Key words: Transfersome, protein, heating method, thin-film rehydration, electrophoresis, nanocarrier

Many peptide and protein drugs must be administered only by injection due to instability and low bioavailability via other routes ${ }^{[1,2]}$. One of the most important proteins for medical applications is insulin, used for the treatment of diabetes. The stress and discomfort of multiple daily injections provide the impetus to develop a safe, effective, and non-invasive route for insulin delivery. A variety of approaches have been developed to improve the delivery, stability and bioavailability of insulin by alternative routes of administration. Most delivery strategies for insulin are based on particulate carriers that have been developed to circumvent the barriers of peptide delivery. Liposomes provide a number of excellent advantages when used in drug delivery systems because of their special structure. First, the enclosed vesicles can separate the inner phase from the outer to improve the stability of an encapsulated drug. Second, when loaded into liposomes, drugs that are poorly soluble in water exhibit enhanced bioavailability ${ }^{[3,4]}$. In addition, a controlled or sustained drug-release profile can be achieved after encapsulation. Furthermore, liposomes possess a high affinity for cells and excellent biodegradability ${ }^{[5,6]}$.
Liposomes are microscopic spherical particles in which membranes, consisting of one or more lipid bilayers, encapsulate a fraction of the solvent in which they are suspended into their interior. Numerous procedures have been developed to prepare liposomes. However, only a few are capable of entrapping large quantities of water-soluble drugs. Drug molecules can be entrapped in liposome vesicles by a variety of methods, including thin-film hydration ${ }^{[7]}$, reversed-phase evaporation ${ }^{[8]}$, solvent injection technique ${ }^{[9]}$, freeze-thaw method ${ }^{[10]}$ and detergent dialysis ${ }^{[11]}$. These are the most commonly used techniques. Techniques such as sonication ${ }^{[12]}$, high pressure extrusion ${ }^{[13]}$ and microfluidization ${ }^{[14]}$ have been employed to help reduce the size of vesicles.

Unfortunately, the conventional preparation methods have some problems. One problem is that the particle

This is an open access article distributed under the terms of the Creative Commons Attribution-NonCommercial-ShareAlike 3.0 License, which allows others to remix, tweak, and build upon the work non-commercially, as long as the author is credited and the new creations are licensed under the identical terms

Accepted 01 December 2018

Revised 27 April 2018

Received 11 August 2017

Indian J Pharm Sci 2019;81(1):110-116 
size of liposomes is too large. The amount of organic solvent remaining in the final product is also a serious issue that not only affects the stability of a protein drug but also affects clinical treatment outcomes. Sterilization of liposomal preparations can also be a problem. In some procedures, careful monitoring is needed, and this may influence the reproducibility of some techniques ${ }^{[15,16]}$. Additionally, these traditional techniques have relative low drug encapsulation in conventional liposomes; previous researches indicated that is less than $45 \%{ }^{[4,17,18]}$.

These traditional techniques require several hours or many days to complete the manufacturing process. Mozafari ${ }^{[19]}$ describes a very fast method to produce liposomes with excellent results in terms of stability and entrapment efficiency. This method involves hydration of the liposome components in an aqueous medium followed by heating the components, which has been shown to produce liposomes that have incorporated drugs efficiently ${ }^{[20,21]}$. Various strategies have been used during preparation to improve encapsulation efficiency. However, changes in hydration media have been suggested to enhance the encapsulation efficiency of peptide drugs, for that reason sucrose $10 \%$ was used as hydrating media ${ }^{[17]}$.

For delivery applications, liposomal formulations should have high entrapment efficiencies, narrow size distributions, long-term stability and ideal release properties (which vary based on the intended application). Achieving these desired properties requires that the preparation method has the potential to produce liposomes using a wide range of ingredient molecules to promote stability ${ }^{[21]}$. Despite numerous research and development studies on liposomes, only a small number of liposomal products have been approved for human use ${ }^{[1,4]}$. This is due to reasons including toxicity of some liposomal formulations, low entrapment of molecules and compounds into liposomes, instability of the liposomal carriers, and high cost of liposome production, especially on large scales $^{[15,16]}$.

Considering the above information, the aim of this work was to determine the physical and chemical differences between ultra-flexible liposomes loaded with insulin obtained by two preparation methods: a very traditional and simple method of thin-film rehydration and the heating method, a relatively new and fast technique. The results of this study aim to show the efficiency of both methods in terms of encapsulation percent, size distribution and stability of the particles.

\section{MATERIALS AND METHODS}

Human recombinant insulin (Pisa Agropecuaria laboratory, Mexico), soy L- $\alpha$ - phosphatidylcholine (PC, Sigma Aldrich, Mexico), sodium cholate (SC, Sigma Aldrich, Mexico) and cholesterol (Sigma Aldrich, Mexico) were acquired from the manufacturing companies. Sucrose $(>99.5 \%)$ was obtained from Sigma Aldrich (Mexico), absolute ethylic alcohol (99.9 \%) was obtained from J.T. Baker (Mexico), and methanol and chloroform were obtained from ACS Fermont (Mexico).

\section{Ultra-flexible liposomes:}

Lipid structures (LS) were obtained from four different proportions of phospholipids using soy L- $\alpha-\mathrm{PC}, \mathrm{SC}$ and cholesterol in the following percent ratios: LS1 (PC $94 \%$, SC $6 \%$ and cholesterol in a 2:3 ratio (PC+SC):(cholesterol)), LS2 (PC $94 \%$, SC $6 \%$ ), LS3 (PC $88 \%$, SC $12 \%$ ), and LS4 (PC $85 \%$, SC $15 \%$ ). All formulations were loaded with human recombinant insulin at a $15 \mathrm{IU} / \mathrm{ml}$ formula concentration. Ultraflexible liposomes were manufactured employing two techniques, the thin-film rehydration method ${ }^{[17,22]}$ and the heating method ${ }^{[19-21]}$, with modification in the rehydrating agent according Park et al. ${ }^{[17]}$.

\section{Thin-film rehydration method:}

Phospholipids were dissolved and mixed in an organic solvent to ensure a homogeneous mixture of lipids. This process was carried out using ethanol and a chloroform/ methanol mixture. Once the lipids were thoroughly mixed in the organic solvent, the solvent was removed to yield a lipid film. The organic phase was eliminated by rotoevaporation (Büchi 461 Rotavapor) at $40^{\circ}$ under a vacuum for $8 \mathrm{~h}$ at $500 \mathrm{mmHg}$ (Rocker 300 vacuum pump). The resulting thin film of phospholipids was hydrated with a $10 \%$ sucrose and insulin solution and placed in the rotary evaporator at the same conditions for $1 \mathrm{~h}$; better insulin encapsulation and reduction in particle size were reported using sucrose $10 \%$ as the rehydrating agent ${ }^{[17,23]}$. After the formulation was placed at room temperature, the resulting dispersion was placed into a water bath (Civeq $\mathrm{HH}-2$ ) at $40^{\circ}$ for $2 \mathrm{~h}$. Finally, the solution was sonicated in an ultrasonic bath (Elmasonic S $30 \mathrm{H}$, Elma) for $5 \mathrm{~min}$.

\section{Heating method:}

Hydrating solution (10\% sucrose with insulin) was mixed in a ball flask with continuous agitation inside a water bath until the temperature reached $60^{\circ}$. When this 
temperature was reached, phospholipids were added, and immediately air was replaced with a nitrogenous atmosphere to reduce lipid oxidation. The flasks were maintained in the water bath at $60^{\circ}$ with constant agitation for $40 \mathrm{~min}$. Then, the resulting liposomes were placed at room temperature until the temperature reached $25^{\circ}$ before transferring the product into amber glass bottles ${ }^{[19,21]}$.

\section{Ultra-flexible liposome characterization:}

The particle size, size distribution, polydispersity index (PDI) and zeta potential (ZP) of the flexible liposomes were measured using an electrophoretic light scattering spectrophotometer (Zetasizer, Nano Sizer, Malvern). Folder cell was used to obtain the measurements. The system was operated in automeasuring mode. Each measurement was carried out in triplicate.

The encapsulation percent of insulin was calculated by the difference between the amount of nonencapsulated insulin (located in the supernatant after ultracentrifugation) and the total amount insulin that was put into the system. Determination of the amount of insulin was performed by UV-Vis spectroscopy ${ }^{[24]}$. Subsequently, the absorbance of all the samples was measured spectrophotometrically at $269 \mathrm{~nm}$ (Varian Cary 1E, Spectralab Analytical).

Before analysis, the formulations were centrifuged in polycarbonate tubes (Beckman, Quick-seal) using an ultracentrifuge (Beckman Coulter, model XL-100K, Beckman Type 100Ti rotor) at $25000 \mathrm{rpm}$ for $80 \mathrm{~min}$ at $4^{\circ}$ to remove any unencapsulated insulin. Each sample was prepared in triplicate using $2 \mathrm{~g}$ of glycerol and $2 \mathrm{ml}$ of sample ${ }^{[21,25,26]}$. After ultracentrifugation, supernatants were centrifuged in acetonitrile at $3000 \mathrm{rpm}$ for $5 \mathrm{~min}$ to precipitate lipids, and resulting supernatants were measured spectrophotometrically. Additionally, the complete formulations were also centrifuged in acetonitrile at $3000 \mathrm{rpm}$ for $5 \mathrm{~min}$ and absorption of the supernatants was quantified at $269 \mathrm{~nm}$ in a spectrometer ${ }^{[24]}$.

Insulin integrity was estimated using polyacrylamide gel electrophoresis (PAGE; Enduro Power Supplies, Labnet International, Inc.). The gels were visualized, and the protein levels were densitometrically evaluated using the software program Quantity One 4.2 (Bio-Rad Laboratories). Insulin levels were measured in triplicate after incubation at $4^{\circ}, 60^{\circ}$ and $100^{\circ}$ temperature.

Stability studies were conducted using native PAGE using a nondenaturing gel $(7.5 \%)$, and visualization was performed using a cytometry gel documentation system $^{[27]}$. The materials used to carry out PAGE were a $30 \%$ acrylamide solution, a separating gel buffer (1.5 M Tris hydrochloride of $\mathrm{pH}$ 8.8), an ammonium persulfate solution (used as an initiator solution), an electrode buffer, a sample loading buffer solution, a staining solution, a destaining solution and a resolving gel $(7.5 \% \text { acrylamide })^{[27]}$.

Gels require approximately 20 min to solidify. Within this time, the gel solution was poured between glass plates and a comb was introduced to make the protein loading wells. The wells were then loaded with samples containing $1 \mu \mathrm{l}$ of a drug solution and $25 \mu \mathrm{l}$ of the sample loading buffer. Then, the glass plates containing the loaded gel were placed into the electrophoresis system and an electric potential was applied. It took approximately $30 \mathrm{~min}$ to run the insulin bands through the gel. The bands of insulin separated by electrophoresis were quantified by cytometry software.

\section{Differential scanning calorimetric (DSC) analysis:}

Data collection was performed using a VP-DSC differential scanning microcalorimeter (MicroCal Incorporated, LLC, Northampton, MA). Insulin thermograms were acquired in triplicate using $7 \mathrm{mM}$ phosphate buffer and a temperature range of $20^{\circ}$ to $100^{\circ}$ and a scan rate of $1 \% \mathrm{~min}$. Data analysis was carried out using Origin 7.0 software (OriginLab, Northampton, MA).

\section{Statistical analysis:}

Data were expressed as the mean \pm standard deviation. Data normality was determined by the Shapiro-Wilk test. The mean differences between groups were analysed using a two-way ANOVA where factor A was the preparation technique with two levels, and factor $\mathrm{B}$ was the formulation with four levels. The differences between means were reported by Tukey's test. All the results were obtained using statistical software (IBM SPSS statistics 20).

\section{RESULTS AND DISCUSSION}

Particle size and size distribution are summarized in Table 1. The values are expressed as an intensity distribution. The intensity distribution describes how much light was scattered by the particles in the different size bins ${ }^{[28,29]}$. All flexible liposome formulations obtained by the thin-film rehydration method showed three groups of particles sizes, while formulations LS2 
and LS3 obtained by the heating method showed two groups of particle sizes. The preparations obtained by the heating method showed a smaller size compared to those prepared by the thin-film rehydration method. The predominant size of the formulations prepared by the heating method was greater than $600 \mathrm{~nm}$, while the size of those prepared by the thin-film rehydration method was greater than $800 \mathrm{~nm}$.

Statistical analysis shows an interaction between the preparation method and the formulation in the $\mathrm{ZP}$ and PDI $(p<0.05)$. The PDI, ZP and encapsulation percent show significant differences between the means of preparation methods and formulations $(\mathrm{p}<0.05)$.

The PDI means were statistically equal for LS1 and LS2 in the thin-film rehydration method and equal for LS2, LS3 and LS4 in the heating method $(p<0.05)$. The results are summarized in Table 2. All the samples show heterogeneous distribution, except for LS1 and LS2 in the thin-film rehydration method, which shows a low PDI value. PDI values greater than 0.5 indicates poor sample uniformity or polysdispersion ${ }^{[30]}$.

The ZP means were significantly different for all formulations, and all formulations except for LS4 showed adequate values in the heating method $(p<0.05)$, results are summarized in Table 2 . The encapsulation percent of LS1 was statistically equivalent to LS2 and the encapsulation percent of LS3 was statistically equivalent to LS4 for both techniques $(p<0.05)$. However, the heating method showed a better percent of insulin encapsulation compared with the same formulation prepared by the thin-film rehydration method. The results were expressed as the mean \pm SD in Table 3.
DSC thermograms of insulin showed a sharp endothermic peak at $85^{\circ}$, which corresponded to its melting point. Table 4 shows the results of the cytometry of the electrophoretic bands; all the measurements were statistically equivalent for the three samples $(p<0.05)$, which indicated that insulin remains completely integrated before and after the preparation of the liposomes.

Insulin has a very low bioavailability when administered by alternative routes due to its low permeability and instability to enzymatic breakdown and $\mathrm{pH}$ changes. Liposomes can provide a protective layer for insulin molecules, and some studies indicate that drug molecules in liposome formulations can penetrate cell membranes more effectively ${ }^{[2,3]}$.

The particle size of the liposomes is the most important factor, as large particles tend to be excreted out of the body at a much faster rate than smaller ones; conversely, very small particles will remain in the body for a longer period, which may cause toxicity ${ }^{[28]}$. The PDI gives information on the particle size distribution, and all the formulations tested showed high polydispersity values, except LS1 and LS2, which showed moderate polydispersity. This characteristic could affect the stability of the system in terms of agglutination of particles $^{[29]}$.

The intensity distribution is naturally weighted according to the scattering intensity of each particle fraction or family. The intensity distribution can be somewhat misleading, in that a small amount of aggregation/agglomeration or the presence of a larger particle species can dominate the distribution. However, this distribution can be used as a sensitive detector for

\section{TABLE 1: SIZE DISTRIBUTION OF LIPOSOMES MADE BY HEATING METHOD AND THIN-FILM REHYDRATION} METHOD

\begin{tabular}{ccccccc}
\hline \multicolumn{7}{c}{ Heating method } \\
\hline LS1 & $1007.33 \pm 22.19$ & $51.07 \pm 1.62$ & $4238.00 \pm 231.27$ & $32.90 \pm 1.15$ & $243.00 \pm 35.59$ & $16.07 \pm 2.65$ \\
LS2 & $673.63 \pm 47.85$ & $90.80 \pm 0.46$ & $100.58 \pm 8.81$ & $9.20 \pm 0.46$ & 0.00 & 0.00 \\
LS3 & $611.37 \pm 13.46$ & $85.60 \pm 0.62$ & $123.90 \pm 12.52$ & $14.40 \pm 0.62$ & 0.00 & 0.00 \\
LS4 & $1152.80 \pm 571.58$ & $63.40 \pm 11.69$ & $1662.40 \pm 2472.28$ & $30.23 \pm 13.46$ & $3316.69 \pm 2804.25$ & $6.30 \pm 2.91$ \\
\hline \multicolumn{7}{c}{ Thin-film rehydration method } \\
LS1 & $994.50 \pm 574.36$ & $52.77 \pm 2.18$ & $4077.67 \pm 779.60$ & $31.17 \pm 3.27$ & $233.45 \pm 44.45$ & $16.10 \pm 1.95$ \\
LS2 & $2165.07 \pm 1964.19$ & $66.93 \pm 14.37$ & $364.97 \pm 324.43$ & $28.13 \pm 9.65$ & $52.57 \pm 57.33$ & $4.67 \pm 4.81$ \\
LS3 & $1366.13 \pm 604.49$ & $69.03 \pm 12.17$ & $271.37 \pm 55.22$ & $25.63 \pm 8.15$ & $51.91 \pm 45.89$ & $8.00 \pm 5.03$ \\
LS4 & $859.00 \pm 44.25$ & $63.33 \pm 12.67$ & $1475.00 \pm 2318.39$ & $25.13 \pm 5.80$ & $3636.17 \pm 3051.69$ & $10.80 \pm 7.43$ \\
\hline
\end{tabular}

Size distribution reported in intensity measure obtained by Zetasizer of flexible liposomes preparations loaded with insulin prepared by heating method and thin-film rehydration method 
the presence of large materials in the sample ${ }^{[31]}$. The heating method resulted in smaller particle sizes and narrower distributions of sizes compared with the thinfilm rehydration method.

The ZP of a liposome preparation can help to predict the fate of the liposomes and their therapeutic efficacy in vivo. Additionally, it supports the investigation of the behaviour of the liposomes as influenced by their surface charge. Liposomes in aqueous dispersions tend to aggregate and subsequently fuse when stored if they have lower $\mathrm{ZP} \mathrm{s}^{[28,29]}$. The value of the $\mathrm{ZP}$ demonstrates the stability of a particulate system. It is a measurement of the repulsive forces between the particles. Particles having a $\mathrm{ZP}$ of less than $-30 \mathrm{mV}$ or more than $+30 \mathrm{mV}$ are usually regarded as stable ${ }^{[29]}$. The $\mathrm{ZP}$ values of the LS2 and LS3 formulations from both methods and the LS4 formulation from the heating method were higher than $+30 \mathrm{mV}$, which confirms their acceptable stability, uniformity and size homogeneity in suspension.

The results showed that flexible liposomes prepared by the heating method possessed a higher trapping percent

TABLE 2: POLYDISPERSITY INDEX AND ZETA POTENTIAL

\begin{tabular}{lcc}
\hline \multicolumn{3}{c}{ Polydispersity index } \\
\hline (Mean \pm SD) & Thin-film rehydration & Heating method \\
\hline LS1 & $0.337 \pm 0.005^{\mathrm{a}}$ & $0.94 \pm 0.031$ \\
LS2 & $0.47 \pm 0.08^{\mathrm{a}}$ & $0.67 \pm 0.07^{\mathrm{b}}$ \\
LS3 & $0.65 \pm 0.05$ & $0.659 \pm 0.09^{\mathrm{b}}$ \\
LS4 & $0.976 \pm 0.03$ & $0.672 \pm 0.17^{\mathrm{b}}$ \\
& Zeta potential & \\
\hline Liposomes & ZP $(\mathrm{mV})$ & ZP $(\mathrm{mV})$ \\
LS1 & $-25.03 \pm 1.01$ & $-26.37 \pm 0.51$ \\
LS2 & $-30.47 \pm 0.91$ & $-46.83 \pm 0.96$ \\
LS3 & $-36.17 \pm 1.10$ & $-70.20 \pm 1.55$ \\
LS4 & $-45.10 \pm 0.78$ & $-1.04 \pm 0.06$
\end{tabular}

Polydispersion index and zeta potential measured by Zetasizer for flexible liposomes loaded with insulin manufactured by two different methods. 'a-b' Within a column, the values with a common superscript letter do not differ significantly $(p<0.05)$ compared with the thin-film rehydration method. The LS1 formulation from both methods showed the lowest insulin encapsulation percent, perhaps because these formulations contain cholesterol. In both techniques, the increase in the proportions of phospholipids and surfactants increased the encapsulation percent, but apparently also increased the size and the heterogeneity of particles. The LS3 and LS4 formulations from the heating method showed encapsulation over $60 \%$. Previous reports indicated that conventional liposomes have encapsulation of $30.30 \pm 2.77^{[18]}$, similar to the encapsulation percent obtained for LS1 and LS2 formulation.

Insulin is subjected to high temperatures during its manufacturing process, which determines the integrity of its structure. The DSC thermograms of insulin indicated a melting point of $85^{\circ}$, and denaturation occurs only if insulin is heated over this temperature. PAGE studies were conducted to confirm the structure of insulin, and it was found to be stable at $4^{\circ}, 60^{\circ}$ and $100^{\circ}$ preparations. PAGE can be used not only as a separation tool as it has been historically used but also as a quantification method for high molecular weight compounds such as proteins if the analysis is accompanied with a gel documentation system ${ }^{[27]}$.

The thin-film rehydration procedure that involves dissolving phospholipids in organic solvents is the most common method for preparation of lipid formulations; however, this method requires at least two days to complete all the procedures. This method is associated with low encapsulation, difficulty of scaling up and a heterogeneous size distribution ${ }^{[16,22]}$. All formulations obtained by the thin-film rehydration method showed a lower encapsulation percent, polydispersity, greater particle size and distribution compared with the heating method.

The heating method ${ }^{[19]}$ is a model technique for fast and simple production of lipid vesicles. Most of the phospholipid molecules employed as liposomal

\section{TABLE 3: ENCAPSULATION PERCENTAGE}

\begin{tabular}{ccccc}
\hline$($ Mean \pm SD $)$ & \multicolumn{2}{c}{ Thin-film rehydration } & \multicolumn{2}{c}{ Heating Method } \\
\hline Liposomes & \% Encapsulated & \% Non-encapsulated & \% Encapsulated & \% Non-encapsulated \\
\hline LS1 & $29.82 \pm 2.0017^{\mathrm{a}}$ & $70.18 \pm 2.00082$ & $35.77 \pm 5.34^{\mathrm{a}}$ & $64.23 \pm 5.34$ \\
LS2 & $36.661 \pm 2.002^{\mathrm{a}}$ & $63.3 \pm 2.004$ & $40.89 \pm 2^{\mathrm{a}}$ & $59.10 \pm 2.0028$ \\
LS3 & $51.25 \pm 3.66^{\mathrm{b}}$ & $48.74 \pm 3.669$ & $61.26 \pm 2.67^{\mathrm{b}}$ & $38.73 \pm 2.67$ \\
LS4 & $57.21 \pm 6.52^{\mathrm{b}}$ & $42.78 \pm 6.519$ & $65.83 \pm 2.62^{\mathrm{b}}$ & $34.16 \pm 2.62$ \\
\hline
\end{tabular}

Encapsulation percentage of insulin was obtained by spectrometer measurement after ultracentrifugation separation of flexible liposomes made by two methods. 'a-b' Within a column, the values with a common superscript letter do not differ significantly $(p<0.05)$ 
TABLE 4: CYTOMETRY OF ELECTROPHORETIC BANDS

\begin{tabular}{cccc}
\hline UAD & Insulin $4^{\circ}$ & Insulin $60^{\circ}$ & Insulin $100^{\circ}$ \\
\hline Mean & $13691.2^{\mathrm{a}}$ & $11357.57^{\mathrm{a}}$ & $9242.064^{\mathrm{a}}$ \\
\pm SD & 1964.62 & 1398.16 & 1126.94 \\
\hline
\end{tabular}

Cytometry of electrophoretic bands of insulin exposed to $4^{\circ}, 60^{\circ}$ and $100^{\circ}$. 'a-b' Values within a row with no common superscript differ significantly $(\mathrm{p}<0.05)$. UAD: cytometry units

constituents have transition temperatures below $60^{\circ}$; thus, in preparations lacking cholesterol, the temperature utilized in this method is adequate to form particles capable of encapsulating a drug ${ }^{[19,21]}$. Moreover, this technique avoids the use of organic solvents, reducing toxicity without affecting the stability or the clinical response. Most of the formulations manufactured by this technique have shown better results in terms of size, encapsulation and stability ${ }^{[21]}$. In addition, the heating method is economical and capable of manufacturing insulin-encapsulated liposomes with a superior dispersity and stability, using a simple and fast protocol in comparison with other traditional methods.

In conclusion, ultra-flexible liposomes obtained by the heating method showed smaller size, relatively better homogeneity, adequate surface charge, and better encapsulation percent compared with formulations manufactured by the thin-film rehydration method and traditional liposomes. The temperature used in this method does not affect the stability of insulin. Moreover, the heating method is a faster, simpler and cheaper process to obtain adequately flexible liposomes successfully loaded with insulin.

\section{Acknowledgements:}

Authors acknowledge the financial support from PAPIIT (Programa de Apoyo a Proyectos de Investigación e Innovación Tecnológica, $\mathrm{N}^{\mathrm{o}}$ IT202317). Additional recognition goes to Dr. Jose Pedraza Chaverri from the Chemistry Department of the Universidad Nacional Autónoma de México, for his support in PAGE procedures. Authors thank Dr. Xelhua Elihut, Marcos Benitez and Dr. Miguel Antonio Costas Basín, researchers from the Chemistry Department, for their support in the DSC technique analysis.

\section{Conflict of interest:}

The authors report no conflicts of interest in this work.

\section{Financial assistance and scholarship:}

Nil.

\section{REFERENCES}

1. Owens D, Zinman B, Bolli G. Alternative routes of insulin delivery. Diabet Med 2003;20:886-98.

2. Iwanaga K, Ono S, Narioka K, Morimoto K, Kakemi M, Yamashita $\mathrm{S}$, et al. Oral delivery of insulin by using surface coating liposomes: Improvement of stability of insulin in GI tract. Int J Pharm 1997;157:73-80.

3. Patel HM, Ryman BE. Oral administration of insulin by encapsulation within liposomes. FEBS Lett 1976;62(1):60-2.

4. Khafagy el-S, Morishita M, Onuki Y, Takayama K. Current challenges in non-invasive insulin delivery systems: A comparative review. Adv Drug Deliver Rev 2007;59(15):152146.

5. Spangler RS. Insulin administration via liposomes. Diabetes Care 1990;13(9):911-22.

6. Sipai Altaf Bhai M, Vandana D, Mamatha Y, Prasanth V. Liposomes: An overview. J Pharm Sci Innov 2012;1(1):13-21.

7. Bangham AD. Properties and uses of lipid vesicles: an overview. Ann N Y Acad Sci 1978;308:2-7.

8. Szoka Jr F, Papahadjopoulos D. Procedure for preparation of liposomes with large internal aqueous space and high capture by reverse-phase evaporation. Proc Natl Acad Sci U S A 1978;75(9):4194-8.

9. Deamer DW. Preparation and properties of ether-injection liposomes. Ann N Y Acad Sci 1978;308:250-8.

10. Pick UI. Liposomes with a large trapping capacity prepared by freezing and thawing of sonicated phospholipid mixtures. Arch Biochem Biophys 1981;212:186-94.

11. Zumbuehl O, Weder HG. Liposomes of controllable size in the range of 40 to $180 \mathrm{~nm}$ by defined dialysis of lipid/detergent mixed micelles. Biochim Biophys Acta 1981;640:252-62.

12. Saunders L, Perrin J, Gammack D. Ultrasonic irradiation of some phospholipid sols. J Pharm Pharmacol 1962;14:567-72.

13. Hope MJ, Bally MB, Webb G, Cullis PR. Production of large unilamellar vesicles by a rapid extrusion procedure. Characterization of size distribution, trapped volume and ability to maintain a membrane potential. Biochim Biophys Acta 1985;812:55-65.

14. Pradhan P, Guan J, Lu D, Wang PG, Lee LJ, Lee RJ. A facile microfluidic method for production of liposomes. Anticancer Res 2008;28:943-7.

15. Popovska O, Simonovska J, Kavrakovski Z, Rafajlovsk V. An Overview: Methods for Preparation and Characterization of Liposomes as Drug Delivery Systems. Int J Pharm Phytopharmacol Res 2013;3(3):182-9.

16. Huang Z, Li X, Zhang T, Song Y, She Z, Li J, et al. Progress involving new techniques for liposome preparation. Asian J Pharm Sci 2014;9(4):176-82.

17. Park SJ, Choi SG, Davaa E, Park JS. Encapsulation enhancement and stabilization of insulin in cationic liposomes. Int J Pharm 2011;415:267-72.

18. Zhang N, Ping QN, Huang GH, Xu WF. Investigation of lectinmodified insulin liposomes as carriers for oral administration. Int J Pharm 2005;294:247-59.

19. Mozafari MR. Liposomes: an overview of manufacturing techniques. Cell Mol Biol Lett 2005;10(4):711-9. 


\section{www.ijpsonline.com}

20. Mozafari MR, Reed CJ, Rostron C. Cytotoxicity evaluation of anionic nanoliposomes and nanolipoplexes prepared by the heating method without employing volatile solvents and detergents. Pharmazie 2007;62:205-9.

21. Mozafari MR. Nanoliposomes: Preparation and Analysis. In: Volkmar W, editor. Liposomes, Methods and Protocols. Vol. 1: Pharmaceutical Nanocarriers. 1st ed. Arizona: Human Press; 2010.

22. Bangham AD, Standish MM, Watkins JC. The action of steroids and streptolysin $\mathrm{S}$ on the permeability of phospholipid structures to cations. J Mol Biol 1965;13:253-9.

23. Vandana M, Sahoo SK. Optimization of physicochemical parameters influencing the fabrication of protein-loaded chitosan nanoparticles. Nanomedicine 2009;4:773-85.

24. Royatvand S, Fallah Hoseini H, Ezzatpanah H, Sekehchi M. Determination of Insulin Concentration in Camel Milk Using Ultra Violet -Visible Absorption Spectroscopy. J Food Bioscience Tech 2013;3:53-60.

25. Janes KA, Alonso MJ. Depolymerized chitosan nanoparticles for protein delivery: Preparation and characterization. J Appl Polym Sci 2003;88(12):2769-76.
26. Grenha A1, Seijo B, Remuñán-López C. Microencapsulated chitosan nanoparticles for lung protein delivery. Eur J Pharm Sci 2005:25(4-5):427-37.

27. Tippavajhala V, Yamsani M. Electrophoretic estimation of insulin: a novel approach to an older technique. Int J Curr Pharm Res 2010;2(4):19-23.

28. Sonia T, Sharma C. Oral insulin delivery. 1st ed. United Kindogm: Woodhead Publishing; 2014.

29. Malvern. Dynamic light scattering common terms defined [cited 2017 April 20]. Available from: http://www.biophysics. bioc.cam.ac.uk/wp-content/uploads/2011/02/DLS_Terms_ defined_Malvern.pdf.

30. Pereira-Lachataignerais J, Pons R, Panizza P, Courbin L, Rouch J, López O. Study and formation of vesicle systems with low polydispersity index by ultrasound method. Chem Phys Lipids 2006;140(1-2):88-97.

31. Malvern. Size and distribution mesure [cited 2017 April 20]. Available from:http://www.malvern.com/en/support/resourcecenter/technical-notes/TN101104IntensityVolumeNumber. aspx. 